\title{
Optimizing Treatment for Head and Neck Cancers: Recurrent/Metastatic Head and Neck Squamous Cell Carcinoma
}

\author{
Presented by Robert I. Haddad, MD
}

\begin{abstract}
Immunotherapy has changed the game in the treatment of head and neck cancer (HNC). Practice-changing results from the phase III KEYNOTE048 trial led to the approval of pembrolizumab immunotherapy alone or in combination with chemotherapy for the treatment of recurrent/ metastatic HNC in the first-line setting. Testing for combined positive score (CPS) is now part of routine practice, because patients with CPS $\geq 1$ can be started on single-agent immunotherapy in the first-line. Pembrolizumab replaces the "old" standard of care established by the EXTREME study, as trials looking at targets besides immunotherapy have proved "disappointing." Additionally, nivolumab and pembrolizumab are both approved for use in the second-line.

J Natl Compr Canc Netw 2020;18(7.5):982-984 doi: 10.6004/jnccn.2020.5009
\end{abstract}

The biggest change to clinical practice for the first-line treatment of patients with metastatic head and neck cancer (HNC) is a new standard of care, according to Robert I. Haddad, MD, Chief, Head and Neck Oncology Program, and Professor of Medicine, Dana-Farber Cancer Institute. In the KEYNOTE-048 study, ${ }^{1}$ patients on pembrolizumab demonstrated superior overall survival (OS) compared with those on the EXTREME regimen. ${ }^{2}$ Based on these results, the FDA approved pembrolizumab alone or in combination with chemotherapy as new firstline standard-of-care therapies for patients with recurrent/ metastatic HNC squamous cell carcinoma (HNSCC).

"You are now required to get a combined positive score [CPS] on your patient," reported Dr. Haddad at the NCCN 2020 Virtual Annual Conference. "In patients with CPS $\geq 1$, you can start by giving single-agentpembrolizumab, and omit the side effects and toxicities of chemotherapy in these patients. It's important to incorporate this test into your routine practice, just as we now incorporate HPV testing in the oropharyngeal sites." Additionally, both nivolumab and pembrolizumab are now FDA-approved for second-line treatment in recurrent/metastatic HNSCC.

\section{Quality of Life in HNC}

"The quality of life of patients with HNC is important when dealing with this disease," he said. "As we all know, these patients have difficulties with social and sensory function, personal characteristics, and physiological function." Patients with HNC experience a significant amount of pain and fatigue, as well as difficulty eating and swallowing. Many are feeding tube-dependent and are unable to socialize, go to work, or maintain intimacy and sexuality. A large number also struggle with insecurities about their appearance, leading to a significant degree of depression. ${ }^{3-5}$

"Our treatment approaches for patients with recurrent/ metastatic disease is to try to improve these functions as much as possible, and obviously to improve OS and quality of life," he said.

First-Line Treatment in Recurrent/Metastatic HNC According to Dr. Haddad, the choice of treatment for these patients depends on a number of factors, including performance status and other comorbidities (eg, alcohol/ tobacco use, aspiration pneumonia, infection, malnutrition). "Remember, many of these patients are heavy alcohol or tobacco users," he noted. "They could have chronic obstructive pulmonary disease or emphysema. All of these factors have to be taken into account in designing treatment." Other factors that affect the choice of first-line therapy include prior treatments and symptoms, as well as patient preference and logistics. And now, in the age of immunotherapy, the role of biomarkers plays an increasingly important role for certain patients.

EXTREME: The "Old" First-Line Standard of Care "Over the past 15 years, the EXTREME regimen has been the standard of care for treating patients in the first-line setting, but that standard has now changed with the 2019 publication of the KEYNOTE-048 study," said Dr. Haddad. 
The phase III EXTREME study enrolled 442 patients with untreated recurrent or metastatic HNSCC. ${ }^{2}$ Patients were randomized to receive a doublet containing cisplatin or carboplatin +5 -FU or a triplet with the same chemotherapy + cetuximab. Patients with stable disease who received chemotherapy + cetuximab continued to receive cetuximab as maintenance until disease progression or unacceptable toxic effects. The study met its primary endpoint of OS, demonstrating an improvement of almost 3 months in favor of the triplet. The response rate was also augmented with the use of cetuximab: $35 \%$ with cetuximab + platinum/5-FU versus $18 \%$ with platinum/5-FU. But then came immunotherapy.

\section{The New First-Line Standard of Care}

The randomized open-label KEYNOTE-048 study demonstrated the superiority of pembrolizumab, alone or in combination, over the EXTREME regimen, ${ }^{1}$ leading to the FDA approval of pembrolizumab + platinum-based chemotherapy and pembrolizumab monotherapy as new first-line standard-of-care therapies for the treatment of patients with recurrent/metastatic HNC.

The 3-arm trial compared pembrolizumab monotherapy versus pembrolizumab in combination with chemotherapy and the EXTREME regimen versus standardof-care with the EXTREME regimen. Patients were enrolled who had locally advanced metastatic HNC with good performance status (ECOG 0 or 1 ) and who were incurable by local therapies. Patients had known p16 status in the oropharynx and had provided tissue for PD-L1 testing. A total of 882 participants from 200 sites in 37 countries were randomized 1:1:1 to receive pembrolizumab alone $(n=301)$, pembrolizumab + chemotherapy $(n=281)$, or cetuximab + chemotherapy $(n=300)$. Most of the study patients $(85 \%)$ had a CPS $\geq 1$ and $43 \%$ had CPS $\geq 20$. Approximately $20 \%$ of patients across all arms had HPV-related disease. In patients with a high CPS $\geq 20$, pembrolizumab + chemotherapy led to a statistically significant improvement in OS, with a median OS of 14.7 versus 11.0 months with the EXTREME regimen $(P=.0004)$. In most patients with a CPS $\geq 1$, OS was also significantly better with pembrolizumab + chemotherapy versus EXTREME (13.6 vs 10.4 months; $P<.0001$ ). Other than CPS scores, OS remained significantly better with pembrolizumab in the total patient population: 13.0 months with pembrolizumab + chemotherapy versus 10.7 months with the EXTREME regimen.

Because toxicity is enhanced in chemotherapycontaining regimens, the hope was that pembrolizumab could hold its own as a single-agent versus chemotherapy + cetuximab, which it did. In all patients, OS in the pembrolizumab monotherapy arm was 11.5 months versus 10.7 months for those in the EXTREME arm, although the difference was not statistically significant $(P=.0199)$.
OS observed in patients according to their CPS scores is "very important to clinical practice," claimed Dr. Haddad. In the patients with a CPS $\geq 20$, median OS was 14.8 months with pembrolizumab versus 10.7 months for chemotherapy + cetuximab, a statistically significant benefit in favor of single-agent pembrolizumab. "More importantly, when you look at the $85 \%$ of patients on this trial who had a CPS $\geq 1$, pembrolizumab also performed better than chemotherapy + cetuximab," he said. Median OS was 12.3 months with immunotherapy versus 10.3 months for EXTREME. "We saw improvements in survival for the single-agent pembrolizumab arm, which will obviously have less toxicity than a triplet of platinum +5 -FU + cetuximab," he added.

The response rate seen with single-agent pembrolizumab was lower than that seen with the EXTREME regimen, although the duration of response was substantially longer with pembrolizumab. Overall response rates were $16.9 \%$ and $36 \%$ with pembrolizumab and EXTREME, respectively. "But remember that the benefit of immunotherapy in oncology does not appear to be in the response rate, but rather in the improvement in OS, which is obviously what's important for these patients," he noted.

Patients with a CPS score $\geq 1$ now have the option of initiating treatment on single-agent pembrolizumab. "This is an important change in practice," he said. "The EXTREME regimen is the old standard of care; KEYNOTE048 now represents the new standard of care for treating patients with recurrent/metastatic HNC."

\section{Other Research Not So Promising}

According to Dr. Haddad, other research looking at targets besides immunotherapy has been "disappointing."

A long-running phase III trial of chemotherapy with or without bevacizumab in patients with recurrent/metastatic HNSCC showed no statistically significant improvement in the primary endpoint of OS with the addition of bevacizumab, although progression-free survival was

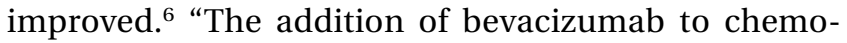
therapy is not superior to chemotherapy alone from an OS perspective," he said. "Because of this, the use of bevacizumab in HNC is not recommended."

\section{Second-Line Immunotherapy in HNC}

"If for some reason you're using chemotherapy first, either because you have a patient with PD-L1-negative disease or who is not tolerating immunotherapy, there are second-line immunotherapy treatment options for patients with HNC," said Dr. Haddad.

Two immunotherapy drugs are now approved for the treatment of recurrent/metastatic HNC in the secondline setting. Nivolumab was approved based on results 
from the phase III CheckMate 141 trial, in which immunotherapy demonstrated a significant improvement in median OS compared with investigator's choice of single-agent chemotherapy (7.5 vs 5.1 months; hazard ratio, $0.70 ; P=.0101) .^{7}$ Pembrolizumab demonstrated similar results in the second-line setting in the KEYNOTE-040 study. ${ }^{8}$

\section{The Future of Treatment}

"The issue we're faced with right now is, if almost all patients are now receiving pembrolizumab with or without chemotherapy in the first line, then the role of immunotherapy in the second line is going to become less and less relevant," said Dr. Haddad. As a result, research in the second-line setting is shifting focus to combination therapies with targets such as PI3K inhibitors, VEGF inhibitors, or chemotherapy. "Depending on what you used in the first line, you might be looking into going back to chemotherapy in the second line, or enrolling patients on therapeutic clinical trials," he added.

The ongoing phase III CheckMate 651 study is targeting the PD-1 and CTLA-4 pathways, comparing combination nivolumab + ipilimumab to the EXTREME regimen as first-line treatment in patients with recurrent/metastatic HNSCC (ClinicalTrials.gov identifier: NCT02741570). Patients with PD-L1-positive tumors appear to respond better to PD-1 inhibitors, he added, but in patients with PD-L1-negative tumors, remember that chemotherapy remains an option.

Disclosures: Dr. Haddad has disclosed that he has received grant/research support from AstraZeneca Pharmaceuticals LP, Bristol-Myers Squibb Company, Genentech, Inc., Merck \& Co., Inc., Loxo Onocology, and Pfizer Inc.; and that he has received consulting fees from AstraZeneca Pharmaceuticals LP, Bristol-Myers Squibb Company, Celgene Corporation, Eisai Inc., Genentech, Inc., Merck \& Co., Inc., Loxo Onocology, and Pfizer Inc.

Correspondence: Robert I. Haddad, MD, Dana-Farber Cancer Institute, 450 Brookline Avenue, D2-126, Boston, MA 02215.

Email: robert_haddad@dfci.harvard.edu

\section{References}

1. Burtness B, Harrington KJ, Greil R, et al. Pembrolizumab Alone or with chemotherapy versus cetuximab with chemotherapy for recurrent or metastatic squamous cell carcinoma of the head and neck (KEYNOTE-048) a randomised, open-label, phase 3 study. Lancet 2019;394:1915-1928.

2. Vermorken JB, Mesia R, Rivera F, et al. Platinum-based chemotherapy plus cetuximab in head and neck cancer. N Engl J Med 2008;359: 1116-1127.

3. Gritz ER, Carmack CL, de Moor C, et al. First year after head and neck cancer: quality of life. J Clin Oncol 1999;17:352-360.

4. Curran D, Giralt J, Harari PM, et al. Quality of life in head and neck cancer patients after treatment with high-dose radiotherapy alone or in combination with cetuximab. J Clin Oncol 2007;25:2191-2197.

5. Rogers SN, Heseltine N, Flexen J, et al. Structured review of papers reporting specific functions in patients with cancer of the head and neck: 2006-2013. Br J Oral Maxillofac Surg 2016;54:e45-51

6. Athanassios A, Li S, Savvides P, et al. Phase III randomized trial of chemotherapy with or without bevacizumab in patients with recurrent or metastatic head and neck cancer. J Clin Oncol 2019;37:3266-3274.

7. Ferris RL, Blumenschein G, Fayette J, et al. Nivolumab for recurrent squamouscell carcinoma of the head and neck. N Engl J Med 2016;375:1856-1867.

8. Cohen EEW, Soulières D, Le Tourneau C, et al. Pembrolizumab versus methotrexate, docetaxel, or cetuximab for recurrent or metastatic headand-neck squamous cell carcinoma (KEYNOTE-040): a randomised, openlabel, phase 3 study. Lancet 2019;393:156-167. 